\title{
ADVANCED LIFE SUPPORT
}

\author{
Revised recommendations of the Resuscitation Council (UK)
}

D A Chamberlain

The first guidelines for advanced life support promoted by the Resuscitation Council of the United Kingdom were published in 1984. The council adopted a policy of revising and updating them every five years so that policies were kept in line with current research. The new recommendations, together with those for basic life support, are now available. The advanced guidelines are summarised in a chart that shows algorithms for treating different modes of cardiac arrest, together with brief advice on the general conduct of resuscitation. This article draws attention to the major modifications in the algorithms.

Since 1984 cooperation has increased greatly with many other European groups that have an interest in cardiopulmonary resuscitation. In particular, we have liaised closely with colleagues

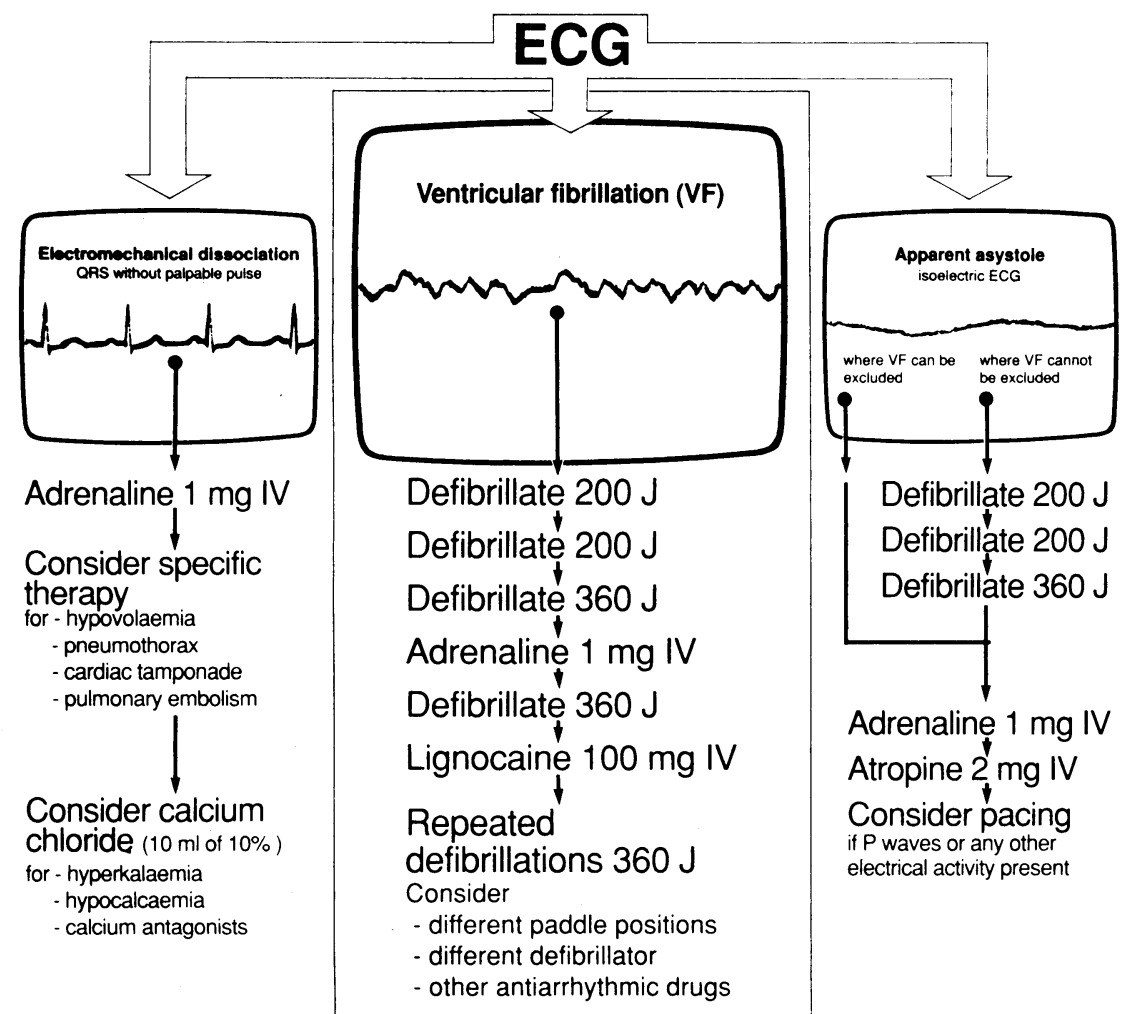

Continue CPR for up to 2 min. after each drug. Do not interrupt CPR for more than 10 sec., except for defibrillation.

If an I.V. line cannot be established, consider giving double doses of adrenaline, lignocaine or atropine via an endotracheal tube.

\begin{tabular}{|ll}
\hline PROLONGED RESUSCITATION: & POST RESUSCITATION CARE \\
$\begin{array}{l}\text { Give } 1 \mathrm{mg} \text { adrenaline IV every } 5 \text { minutes. } \\
\text { Consider } 50 \mathrm{mmol} \text { sodium bicarbonate }(50 \mathrm{ml} . \text { of } 8.4 \%)\end{array}$ & $\begin{array}{l}\text { Check } \\
\text { or according to blood gas results. }\end{array}$ \\
& $\begin{array}{l}\text { - arterial blood gases } \\
\text { - electrolytes } \\
\text { Observe monitor and treat patient } \\
\text { in an intensive care area. }\end{array}$ \\
\hline
\end{tabular}

from Nordic countries. The recommendations for 1989-and the chart - are substantially the same for the United Kingdom, Sweden, Denmark, Norway, and Finland. We expect that the 1994 recommendations will represent a consensus from a larger group of European countries.

The use of the precordial thump has been reintroduced but only for witnessed or monitored arrests, in line with published evidence of its efficacy for pulseless ventricular tachycardia or ventricular fibrillation of very recent onset. The risk of exacerbating a ventricular arrhythmia is recognised, but the likelihood of harm is small when the arrest has been witnessed. Moreover, a proarrhythmic effect is possible with all successful interventions, and the manoeuvre takes little time to execute.

\section{Defibrillation}

No modifications have been made to the recommendations for defibrillation, except in so far as the energy levels now conform to the existing standards for the calibration of modern defibrillators in calculated delivered energy. Some equipment still in use will be calibrated in terms of stored energy and may have settings that correspond only roughly to the figures that are now appropriate.

\section{Order of drugs}

An important change has been made in the order of the drugs that should be given if ventricular fibrillation fails to respond to the first three shocks. Adrenaline has replaced lignocaine as the first pharmacological agent. Lignocaine is of proved efficacy in treating ventricular tachycardia and in the prophylaxis of ventricular fibrillation, and by extrapolation many believe that the drug must be of value as an adjunct to defibrillation. This notion has, however, never been supported by any convincing clinical evidence; moreover, experimental observations suggest that fibrillation becomes more rather than less refractory to electrical treatment after the administration of lignocaine. The reader may well protest that instances do occur in which defibrillation is successful only after lignocaine has been given. We now understand, however, that the concept of a defibrillation threshold is simplistic. Many factors determine whether or not a shock will be successful, and some of these (perhaps, for example, the vector of the waveform) will vary from moment to moment. Thus the fourth shock in a series may well 
defibrillate when others have failed quite irrespective of drug treatment. In the absence of relevant clinical data we decided to follow the experimental evidence, though we acknowledge that the substrate for the arrhythmia is different in clinical and experimental settings.

While the evidence to commend adrenaline as an adjunct to defibrillation is no better than that for lignocaine, it does have one other attribute that led us to include it earlier in the algorithm. Animal experiments offer convincing evidence that adrenaline increases the cerebral circulation during basic life support. The main purpose of adrenaline at this point therefore is to maintain cerebral perfusion during a prolonged resuscitation attempt. We make no claim that it enhances the efficacy of a direct current shock, though many believe that this can occur in some circumstances.

The use of sodium bicarbonate is no longer encouraged except in prolonged resuscitation. Even then we hope its use will be restricted to victims who are being ventilated efficiently, for otherwise intracellular acidosis is increased rather than diminished. The explanation for the paradox is not new. The carbon dioxide formed by the breakdown of bicarbonate readily crosses cell membranes unless it is removed by respiration. The alkaline moiety, on the other hand, remains predominantly extracellular.

The advice at the end of the defibrillation algorithm has also been modified. Repeated shocks at maximum energy levels are advised if fibrillation is refractory after the initial three shocks and the two drugs (plus the fourth shock) have been administered. We do not suggest a limit to the number of attempts at defibrillation if the rhythm diagnosis is accurate, for the victim is unlikely to have any other chance of survival. Different paddle positions and a change of defibrillator should be considered, however, together with other antiarrhythmic drugs. The chart does not specify the drugs because of differences in availability between the United Kingdom and Scandinavia. In the United Kingdom bretylium tosylate would be a strong contender for this role.

\section{Asystole}

An important change has been introduced in the advice offered for victims of apparent asystole. This electrical disorder has grave prognostic implications, and treatment is generally disappointing, especially in ischaemic heart disease. Errors have occurred owing to the

\section{Consider calcium} chloride $(10 \mathrm{ml}$ of $10 \%)$

for - hyperkalaemia hypocalcaemia calcium antagonists

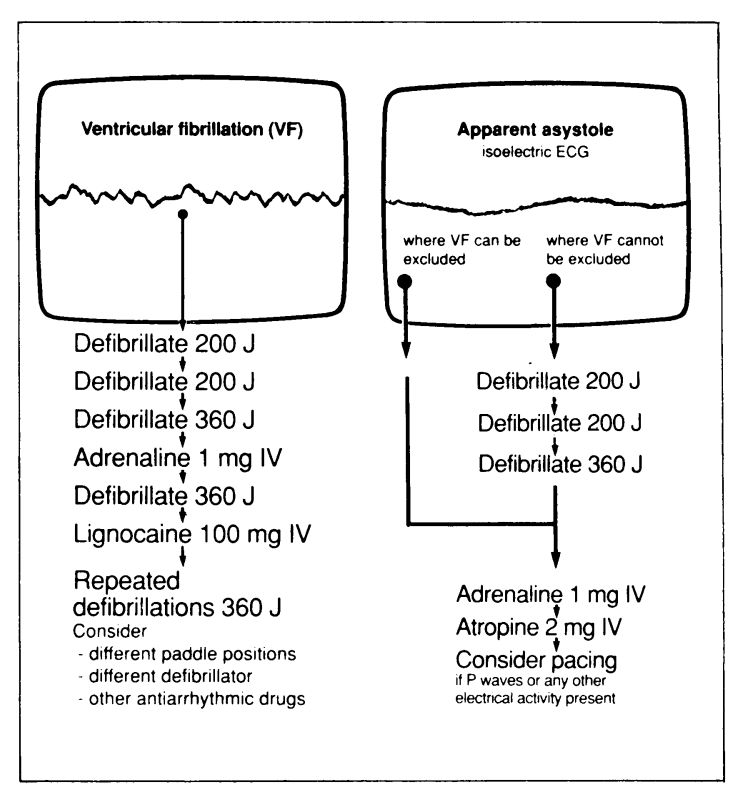

incorrect diagnosis of asystole in victims who are in ventricular fibrillation. This may occur if equipment is faulty, if the gain control of the electrocardiograph has been turned down, if ventricular fibrillation is very fine, or, rarely, if the dominant vector of the waveform is at right angles to the bipolar diagnostic lead. The possibility of incorrect diagnosis should be emphasised lest a very treatable abnormality should remain without proper treatment. If th $e$ rescuer feels that the possibility of fibrillation has not been excluded then three shocks should be administered before the algorithm for true asystole is started. The algorithm itself has also been modified, with adrenaline now having precedence over atropine. Isoprenaline is no longer recommended, and the possibility of using the intracardiac route for adrenaline has been deleted.

\section{Electromechanical dissociation}

We hope that the recommendations for electromechanical dissociation will be considered to be more helpful than before. The possibility of hypovolaemia has now been included, because haemorrhagic shock is an important cause of an impalpable pulse that should not be overlooked. Other causes of electromechanical dissociationapparent or real-have been presented before, but the special circumstances in which calcium chloride has undoubted value have now been listed.

\section{Maintaining resuscitation}

The importance of maintaining cardiopulmonary resuscitation is emphasised: no more than 10 seconds should be permitted for any manoeuvre that demands temporary cessation of chest compression. The endotracheal route is now recognised as being an unreliable route for drug administration and should be used only when an intravenous line cannot be established. Repeated doses of adrenaline and sodium bicarbonate are recommended for prolonged resuscitation. Postresuscitation checks are 
mentioned in the present chart, another minor innovation compared with its predecessor.

We do not claim that the 1989

recommendations represent the permanent truth, but we do believe that they can all be justified by reasonable evidence. We also believe that uniformity in resuscitation guidelines has much to commend it. We hope that our guidelines will be widely endorsed.

Members of Resuscitation Council

P J F Baskett, A J Camm, D A Chamberlain, T Evans, J Fisher, F StC Golden, A J Handley, M Harries, R L Herbert, A K Marsden, A McGowan, A D Milner, A D Redmond, C Robertson, R S Simons, D V Skinner, R A Sleet,
B Steggles, H Tunstall-Pedoe, $\mathrm{R}$ Vincent, $M$ E Ward, D A Zideman.

Basic life support working group

Chairman: A K Marsden. Coopted members: R F Furber, C James, C Moffatt, C C Molloy, D O'Brien, G Wynne.

Advanced life support working group

Chairman: D A Chamberlain. Coopted members: J Adgey, A R Aitkenhead, D Bennett, T Cripps, S Holmberg,

D Julian, L Mogensen, E Sandøe, D G Waller.

For further information write to: Dr D A Zideman, Department of Anaesthetics, Royal Postgraduate Medical School, Hammersmith Hospital, DuCane Road, London W12 OHS. A revised edition of $A B C$ of Resuscitation, incorporating these revised guidelines, will be published in the autumn.

\title{
NHS Review
}

\section{Improving clinical effectiveness in the NHS: an alternative to the white paper}

\author{
Lois Quam
}

The government's argument in support of its white paper Working for Patients ${ }^{1}$ has relied partly on the lack of any other articulated strategy for reform. No one can quarrel with the need to improve the efficiency or effectiveness of the NHS, but there are distinct alternative strategies for that reform. A clinical effectiveness reform is presented as a sounder and more plausible means for improving the NHS

\section{Fundamental assumptions of the white paper}

The white paper is built on three fundamental assumptions. Firstly, it assumes that price in medical care effectively reflects value and that trading in internal markets would, therefore, increase efficiency despite the absence of supporting theoretical and empirical evidence. ${ }^{2}$ Managers lack the information to purchase value for money because the data do not include measures of the effectiveness of care provided. Price based competition is inevitable because no existing quality measures compete with price in terms of coherency, comprehensiveness, and decision making power.

Secondly, the white paper assumes that patient throughput measures, such as performance indicators, effectively measure health care performance despite the absence of measures of patient outcome. These are pale surrogates for quality of care. The white paper contains a surfeit of input measures and a deficit of output measures.

Thirdly, the white paper truncates the definition of quality of care into one of patient choice. Other dimensions of care are neglected including the quality
Despite the competitive strategy's focus on cost containment medical care inflation in the United States is unrestrained, and the increase in the annual rate of medical care expenditure was higher in the 1980s than the 1970s. The rising costs of administration, which have contributed to increased health care costs in the $1980 \mathrm{~s}$, are incurred by the need to track complex financial transactions and regulate the market. These burgeoning costs in the competitive system have coincided with persistent concerns over the quality of care,${ }^{6}$ low rates of patient satisfaction, and an increasing proportion of the population without health insurance.

This failure to control costs and ensure quality has meant that the competitive strategy has lost many adherents in the United States at the same time as the government has introduced it in the United Kingdom. Because of the fear that price based competition has diminished quality of care in the United States, a new strategy aimed at measuring patient outcomes is being put forward by the medical profession, ${ }^{8}$ the federal government, ${ }^{9}$ and supporters of the competitive market approach. ${ }^{10}$

\section{Alternative reform of the NHS}

A reform of the NHS directed at improving the effectiveness and efficiency of clinical care is an alternative to the white paper.

\section{... research in the United States}

\section{shows the danger inherent in invalid} performance indicators.

The assumptions of this clinical effectiveness reform are in fundamental disagreement with the white paper's proposals. Firstly, this reform assumes that value in medical care must include the quality of patient outcomes. Secondly, performance indicators in medicine are assumed effective only if they measure the process and outcomes in medical care. This argument is well known in industrial quality assurance.

\footnotetext{
of patient outcomes; the extent to which the process of care adheres to the best known medical practice; and protection from substandard practitioners. Moreover, this focus on patient choice in the white paper is baffling because managers will steer patients to facilities with bulk purchase agreements. Money will precede patients rather than follow them.

These white paper assumptions are familiar to American doctors and managers. In 1981 the Reagan administration looked to the market to control costs and quality. ${ }^{3}$ Indeed, some of the same economic advisers participated in the development of both the American and the British competitive strategies. ${ }^{45}$

BrMed f 1989;299:448-50

\section{Bren Road East, \\ Minneapolis, Minnesota \\ United HealthCare \\ Lois Quam, BA, director, \\ Correspondence to: \\ Department, United \\ 55434, USA research and development
Lois Quam,}

Corporation, Minneapolis,
} 